\title{
Administrative Claims Analysis of Utilization and Costs of Care in Health Plan Members With Atopic Dermatitis Who Had Prior Use of a Topical Corticosteroid and Who Initiate Therapy With Pimecrolimus or Tacrolimus
}

\author{
Thomas E. Delea, MSIA; Manjusha Gokhale, MA; Charles Makin, MS; Mohamed A. Hussein, MSCS, MSPh; \\ Julie Vanderpoel, PharmD, MPA; Timothy Sandman, MBA; Jane Chang, MPH; Jennifer Sung, PharmD; \\ Paul Pinkston, PharmD; Douglas Gause, PhD; and J. Mark Jackson, MD
}

\begin{abstract}
BACKGROUND: In the United States, pimecrolimus cream and tacrolimus ointment are approved as second-line therapy for short-term and intermittent noncontinuous long-term treatment of atopic dermatitis (AD) in nonimmunocompromised patients aged 2 years or older who have failed to respond adequately to other topical prescription treatments (e.g., topical corticosteroids), or when those treatments are not advisable; pimecrolimus is indicated for mildto-moderate $A D$ and tacrolimus for moderate-to-severe $A D$. Comparative data on the effects of pimecrolimus versus tacrolimus on AD-related health care utilization and costs among similar patients seen in typical clinical practice are currently unavailable.
\end{abstract}

OBJECTIVE: To compare utilization and costs of AD-related medical care in health plan members with $A D$ who had prior use of a topical corticosteroid and who subsequently initiate therapy with pimecrolimus cream or tacrolimus ointment.

METHODS: This was an observational, retrospective study using an administrative claims database with dates of service from August 1, 2000, through October 31,2003 , and representing approximately 2.5 million members in health maintenance organizations, preferred provider organizations, and Medicare and Medicaid plans mostly located in the cities of Chicago, Kansas City, and Phoenix and in the states of Kentucky, Florida, and Texas. The study sample included all members with 1 or more pharmacy claims for a topical corticosteroid and a diagnosis of AD (International Classification of Diseases, Ninth Revision, Clinical Modification [ICD-9-CM] code 691.XX (excluding 691.0X), or 692.XX (excluding 692.0X-692.8X)] who subsequently had 1 or more pharmacy claims for pimecrolimus or tacrolimus. AD-related utilization and medical care costs (plan payments plus member cost share) over 12 months of follow-up were compared between the pimecrolimus and tacrolimus groups. Because information on disease severity was not available in the administrative claims data, propensity matching was used to control for differences between groups in baseline demographic and clinical characteristics and pretreatment utilization of AD-related medical care services.

RESULTS: Before matching, compared with the tacrolimus group $(n=197)$, members in the pimecrolimus group $(n=197)$ were older (mean age of 38 vs. 32 years, $P=0.022$ ), had fewer topical corticosteroid pharmacy claims (mean 2.08 vs. $3.01, P=0.002$ ), and had fewer grams of corticosteroids dispensed (mean 132 vs. $193, P=0.029$ ) in the 12 months prior to treatment. After matching, there were 157 members in each group with no statistically significant differences in pretreatment characteristics. During the 12-month follow-up period, the mean (median) number of pharmacy claims was 1.8 (1.0) for pimecrolimus versus 2.0 (1.0) for tacrolimus and the mean (median) grams of study medication were $102(60)$ and $105(60)$, respectively. Members in the pimecrolimus group received a lower average number of prescriptions for any topical corticosteroids (1.37 vs. 2.04, $P=0.021)$ and for high-potency topical corticosteroids ( 0.61 vs. $1.04, P=0.023)$ and were less likely to initiate alternative therapy ( $5 \%$ vs. $17 \%, P<0.001)$ or receive antistaphylococcal antibiotics ( $16 \%$ vs. $27 \%, P=0.014$ ). Members in the pimecrolimus group had lower average (median) AD-related expenditures (75\% to $78 \%$ attributable to AD drug cost) compared with matched tacrolimus members (\$263 [\$270] vs. $\$ 361$ [\$398], $P=0.012)$.
CONCLUSIONS: In health plan members with AD who had previously received at least 1 topical corticosteroid prescription, the customary use of pimecrolimus or tacrolimus was 1 to 2 prescriptions in 12 months of followup and only a median of 60 grams of topical medication. The difference in AD-related utilization and costs between pimecrolimus and tacrolimus was small, less than $\$ 100$ per year, but favored pimecrolimus. Further research using validated measures of disease severity to control for potential confounding is needed to confirm the results of this observational study.

KEYWORDS: Atopic dermatitis, Pimecrolimus, Tacrolimus, Retrospective claims analysis, Costs

J Manag Care Pharm. 2007;13(4):349-59

Copyright@ 2007, Academy of Managed Care Pharmacy. All rights reserved.

\section{What is already known about this subject}

- A pooled analysis of 413 adults and 650 pediatric patients participating in 3 randomized studies found that tacrolimus compared with pimecrolimus was more effective based on (1) Investigator Global Atopic Dermatitis Assessment (IGADA), (2) improvement in percentage of total body surface area affected, and (3) improvement in itch scores; tacrolimus and pimecrolimus had comparable tolerability.

\section{What this study adds}

- Using propensity matching to control for differences between groups in baseline demographic and clinical characteristics and pretreatment utilization of AD-related medical care services in the absence of explicit classification of disease severity, the present study suggests that in real-world clinical practice, use of pimecrolimus may be associated with fewer pharmacy claims for topical corticosteroids, less utilization of antistaphylococcal antibiotics, and small but lower total costs of AD-related medical care (75\% attributable to AD drug cost) compared with tacrolimus.

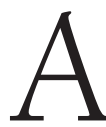
topic dermatitis (AD) is a chronic or relapsing skin disease characterized by itching, redness, and inflammation, with a predilection for the face and extensor surfaces of the extremities in young children and the flexural folds in older children and adults. ${ }^{1}$ Although AD typically presents in infancy 


\section{Administrative Claims Analysis of Utilization and Costs of Care in Health Plan Members With Atopic Dermatitis Who Had Prior Use of a Topical Corticosteroid and Who Initiate Therapy With Pimecrolimus or Tacrolimus}

or early childhood and resolves with advancing age, it may persist into adulthood or commence at that time of life. In industrialized countries, the lifetime prevalence of $\mathrm{AD}$ is $10 \%$ to $20 \% .^{2}$ There is evidence that the incidence of $\mathrm{AD}$ has increased over the past several decades. ${ }^{3} \mathrm{AD}$ has a negative impact on patient quality of life ${ }^{4-6}$ and imposes a substantial economic burden on health care systems as well as on patients and their families. ${ }^{7-11}$

Topical corticosteroids and moisturizing therapy have long been the mainstay of pharmacological treatment for $\mathrm{AD}$. Although effective, prolonged use of steroids may lead to skin atrophy, hyperpigmentation, and, rarely, suppression of the hypothalamic-pituitary-adrenal axis. ${ }^{12-15}$ These effects may require the use of alternative topical agents. ${ }^{16,17}$

Pimecrolimus cream and tacrolimus ointment are topical calcineurin inhibitors that are indicated for the short-term and intermittent long-term treatment of $\mathrm{AD}$ in immunocompetent patients aged 2 years or older who have failed to respond adequately to topical corticosteroids or who have been advised not to take topical corticosteroids (e.g., due to concerns regarding hyperpigmentation on head and neck areas). ${ }^{18}$ Pimecrolimus cream $1 \%$ is indicated for all patients aged 2 years and older. The $0.03 \%$ strength tacrolimus ointment is recommended for children aged 2 to 15 years and the $0.1 \%$ strength is recommended for adults.

Randomized, blinded, vehicle-controlled trials have shown that pimecrolimus and tacrolimus are more effective than placebo vehicle in treatment of AD. ${ }^{19-26}$ They are minimally absorbed and do not produce the adverse effects on the skin that are frequently seen with topical corticosteroids. ${ }^{27}$ While the 2 drugs have a similar mechanism of action, they differ in their pharmacological profiles, with pimecrolimus characterized by less skin permeation and systemic immunoreactions than for tacrolimus. ${ }^{28}$ Tacrolimus was approved by the U.S. Food and Drug Administration (FDA) in December 2000 and became available in the U.S. market in January 2001; pimecrolimus was approved by the FDA in December 2001 and became available in the U.S. market in March 2002. ${ }^{18}$ Importantly, whereas pimecrolimus is indicated for mild-to-moderate $\mathrm{AD},{ }^{29}$ tacrolimus is indicated for moderate-to-severe AD. ${ }^{30}$ The FDA has mandated a warning of a potential cancer risk from longterm use of topical immunosuppressant calcineurin inhibitors on the basis of animal studies, case reports, and mechanism of action, ${ }^{31}$ although a definitive causal link between use of these drugs and cancer has not been established.

In a meta-analysis of 16 noncomparative trials ( 9 tacrolimus and 7 pimecrolimus) involving a total of 5,301 patients (2,106 for tacrolimus and 1,225 for pimecrolimus), Iskedjian and colleagues reported similar clinical success rates for pimecrolimus and tacrolimus. ${ }^{32}$ The populations examined in the trials of pimecrolimus and tacrolimus differed, however, with trials of tacrolimus trials enrolling patients with more severe disease, which may have biased the comparison. ${ }^{32}$ Head-to-head comparative clinical studies of pimecrolimus and tacrolimus have had contradictory findings. In a randomized, investigatorblinded study of pimecrolimus ( $\mathrm{n}=71$ ) and tacrolimus ointment $0.03 \%(n=70)$ in pediatric patients with moderate AD, Kempers and colleagues reported that efficacy based on the Investigator Global Atopic Dermatitis Assessment (IGADA) score was not statistically different between groups. ${ }^{33}$ However, patients receiving pimecrolimus had less frequent adverse skin reactions lasting more than 30 minutes and were more likely to rate ease of application as excellent or very good. ${ }^{33}$

More recently, in a pooled analysis of the results of 3 randomized comparative studies of pimecrolimus versus tacrolimus involving 413 adult and 650 pediatric patients, Paller and colleagues reported that tacrolimus was more effective than pimecrolimus, based on improvement from baseline to 6 weeks or the end of the study in Eczema Area Severity Index (EASI) (52.8\% vs. $39.1 \%$, respectively; $P<0.001$ ), IGADA scores (treatment success $62.1 \%$ vs. $49.8 \%, P<0.001$ ), and percentage of total body surface area affected ( $53.6 \%$ vs. $41.0 \%, P<0.001) .{ }^{34}$ The least squares mean itch scores at baseline were 5.6 in both groups, and tacrolimus patients had lower least squares mean itch scores (2.6) at the end of the study (6 weeks) versus 3.3 for pimecrolimus, $P<0.001$. The safety profiles of the 2 drugs were similar. Although a recent budgetary analysis reported that the addition of pimecrolimus as a treatment would have a minimal impact on AD-related costs for a health plan, ${ }^{35}$ and a recent cost-effectiveness analysis suggested that pimecrolimus is a cost-effective alternative for patients with $\mathrm{AD},{ }^{36}$ neither of these studies considered tacrolimus.

Although results from clinical trials suggest that there may be differences in efficacy, safety, and tolerability of pimecrolimus and tacrolimus, data on the differences in utilization and costs of AD-related medical care for patients receiving these 2 drugs in typical clinical practice are unavailable. We therefore conducted a retrospective observational health insurance claims-based study to compare AD-related utilization costs in health plan members receiving pimecrolimus or tacrolimus within 1 year following first prescription of either of these agents.

\section{Methods}

\section{Study Design}

This was a retrospective observational study comparing the utilization and costs of AD-related medical care among AD patients who had received at least 1 prescription for a topical corticosteroid and who subsequently initiated either pimecrolimus or tacrolimus. Data were obtained from a large administrative claims dataset. Utilization and costs during the 12 months following the first pharmacy claim for pimecrolimus or tacrolimus were compared using propensity score matching to control for differences in demographic characteristics and pretreatment utilization and costs of AD-related medical care. 


\section{Administrative Claims Analysis of Utilization and Costs of Care in Health Plan Members With Atopic Dermatitis \\ Who Had Prior Use of a Topical Corticosteroid and Who Initiate Therapy With Pimecrolimus or Tacrolimus}

\section{Data Source}

Data for this study were obtained from a Health Insurance Portability and Accountability Act (HIPAA)-compliant, limited dataset containing enrollment data as well as facility, professional service, and outpatient pharmacy claims for approximately 2.5 million members enrolled in a health maintenance organization (HMO), preferred provider organization, and Medicare and Medicaid plans mostly located in the cities of Chicago, Kansas City, and Phoenix and in the states of Kentucky, Florida, and Texas. Age, sex, U.S. Census region, and dates of benefit eligibility were generally available for all members. Data available for each outpatient pharmacy claim included the drug dispensed (in National Drug Code [NDC] format), the dispensing date, and the quantity and number of therapy days dispensed. Data available for each facility or professional service claim ("medical claim") included dates of service and International Classification of Diseases, Ninth Revision, Clinical Modification (ICD-9-CM) diagnosis codes; severity of AD was not ascertainable based on diagnosis codes. Facility claims also included ICD-9-CM procedure codes, revenue codes, and discharge status, while professional service claims also include Health Care Common Procedure Coding and Current Procedural Terminology procedure codes. Most claims also included information on paid amounts (plan payment and member cost share). Claims for an individual member were linkable using a unique encrypted patient identifier. This study included claims spanning the period from August 1, 2000, to October 31, 2003 ("study period").

\section{Study Sample}

To identify the study sample, we selected all members in the database with 1 or more pharmacy claims for a topical corticosteroid between August 1, 2000, and October 31, 2002. The date of the first pharmacy claim for a topical corticosteroid was designated the "steroid start date." From these members, we selected those with 1 or more pharmacy claims for pimecrolimus or tacrolimus between August 1, 2001, and October 31, 2002, and after the steroid start date. The date of the first pharmacy claim for either pimecrolimus or tacrolimus was designated the "index date." The 12-month period prior to the index date was designated the "pretreatment period." The 12-month period subsequent to the index date was designated the "follow-up period."

Members meeting the above criteria were then stratified into treatment groups (pimecrolimus or tacrolimus) based on the study medication received on the index date; those with pharmacy claims for both pimecrolimus and tacrolimus on the index date were excluded. Other exclusion criteria were as follows:

1. Fewer than 12 months of continuous eligibility for drug and medical benefits during preindex and postindex periods

2. Less than 1 medical claim with a diagnosis (primary or secondary) of AD (ICD-9-CM 691.XX, excluding 691.0X, or 692.XX, excluding 692.0X-692.8X) during the pretreatment period
3. One or more medical claims with a diagnosis of diaper rash, contact dermatitis, or dermatitis due to substances taken internally (ICD-9-CM 691.0X, 692.0X-692.8X, 693.XX) during the pretreatment period

4. Age less than 2 years as of the index date

Because we required 12 months of continuous enrollment prior to the first prescription for pimecrolimus or tacrolimus, the "washout" period for these drugs was 12 months.

\section{Patient Characteristics}

Information on age, sex, U.S. Census region, and plan type was obtained from enrollment files for each member. Medical claims during the pretreatment period were scanned to identify members with 1 or more encounters for other inflammatory conditions of the skin and subcutaneous tissue (ICD-9-CM 690.XX-698.XX, excluding codes for AD described above), staphylococcal infection of the skin and subcutaneous tissue (ICD-9-CM 041.1X and 680.XX-709.XX), and nonspecific dermatitis (ICD-9-CM 692.9X).

\section{Measures of Utilization and Costs}

For each member, we calculated several measures of utilization and costs during the 12-month pretreatment and followup periods. Utilization measures included the total number of pharmacy claims and grams of low-potency topical corticosteroids (e.g., hydrocortisone cream or ointment $1.0 \%$ or $2.5 \%$, fluocinolone acetonide cream $0.01 \%$ ), medium-potency topical corticosteroids (e.g., triamcinolone acetonide cream or ointment $0.025 \%$ or $0.1 \%$, betamethasone valerate cream or ointment $0.1 \%$ ), and high-potency topical corticosteroids (e.g., clobetasol propionate cream or ointment $0.05 \%$, fluocinonide cream or ointment $0.05 \%$ ) during the pretreatment and follow-up periods. ${ }^{37}$ We also identified members who had pharmacy claims for antihistamines, systemic immunosuppressives, or antistaphylococcal antibiotics during the pretreatment and follow-up periods as well as those who had pharmacy claims for the alternative study medication during the follow-up period (i.e., tacrolimus if in the pimecrolimus group and pimecrolimus if in the tacrolimus group). We also calculated for each member the number of AD-related visits to specialists (allergist, dermatologist, or immunologist) and nonspecialists during the pretreatment and follow-up periods. Visits were classified as AD-related if there was a diagnosis of AD (ICD-9-CM 691.XX excluding 691.0X, or 692.XX excluding 692.0X-692.8X) on the claim.

Costs measures included the cost of AD-related visits (as defined above), the costs of AD-related medications, and total AD-related costs (i.e., the costs of AD-related visits plus the cost of AD-related medications) during the pretreatment and followup periods. AD-related medications included pimecrolimus, tacrolimus, topical corticosteroids, antihistamines, systemic immunosuppressives, and antistaphylococcal antibiotics. The 


\section{Administrative Claims Analysis of Utilization and Costs of Care in Health Plan Members With Atopic Dermatitis Who Had Prior Use of a Topical Corticosteroid and Who Initiate Therapy With Pimecrolimus or Tacrolimus}

cost for each claim was defined as the sum of plan payment and member cost share (i.e., allowable charge). In calculating measures of utilization and costs, office visits occurring on the index date were assigned to the pretreatment period, while pharmacy claims occurring on the index date were assigned to the follow-up period.

\section{Statistical Analyses}

Demographic characteristics, as well as measures of utilization and costs of AD-related medical care during the pretreatment and follow-up periods, were compared between the treatment groups using chi-square tests for categorical variables and the Student $t$ test for continuous variables. To control for differences between groups in baseline characteristics, we also compared measures of utilization and costs during follow-up among propensitymatched members in the pimecrolimus and tacrolimus groups. ${ }^{38,39}$ Propensity scores were calculated for all members by estimating a logistic regression model with treatment group as the dependent variable and selected patient demographic characteristics and pretreatment measures of AD-related utilization as independent variables. The latter included age, sex, region, plan type, pretreatment diagnosis of other inflammatory skin condition, pretreatment claims for antistaphylococcal antibiotics, antihistamines, or systemic immunosuppressive medications, numbers of pretreatment AD-related specialist and generalist visits, and numbers of pretreatment steroid claims. The propensity score for each member was defined as the predicted probability (range: 0.0-1.0) of receiving pimecrolimus conditioned on the observed values of the other characteristics. Matched pairs of pimecrolimus and tacrolimus members were identified using a greedy matching technique so that the maximum difference in propensity score between paired members was $0.1 .{ }^{40}$ Because tacrolimus is available in 2 dosage forms $(0.03 \%$ and $0.1 \%)$ and clinical effectiveness might differ by formulation, we conducted a secondary analysis in which we compared members receiving pimecrolimus with matched members receiving tacrolimus $0.1 \%$. All analyses were conducted using SAS Proprietary Software, Release 9.1 (Cary, NC).

\section{Results}

\section{Study Sample}

We identified 17,341 members who had 1 or more pharmacy claims for a topical corticosteroid between August 1, 2000, and October 31, 2002 (see Figure). Of these, 1,502 had a pharmacy claim for pimecrolimus or tacrolimus between August 1, 2001, and October 31, 2002. These 1,502 members included 832 who had claims for pimecrolimus on the index date and 662 who had claims for tacrolimus on the index date, as well as 8 members who had claims for both tacrolimus and pimecrolimus on the index date and who were therefore excluded from the analysis. In the pimecrolimus group, 496 (60\%) were excluded because they did not have a pharmacy claim for topical steroids prior to

\section{FIGURE Flowchart of Study Subjects}

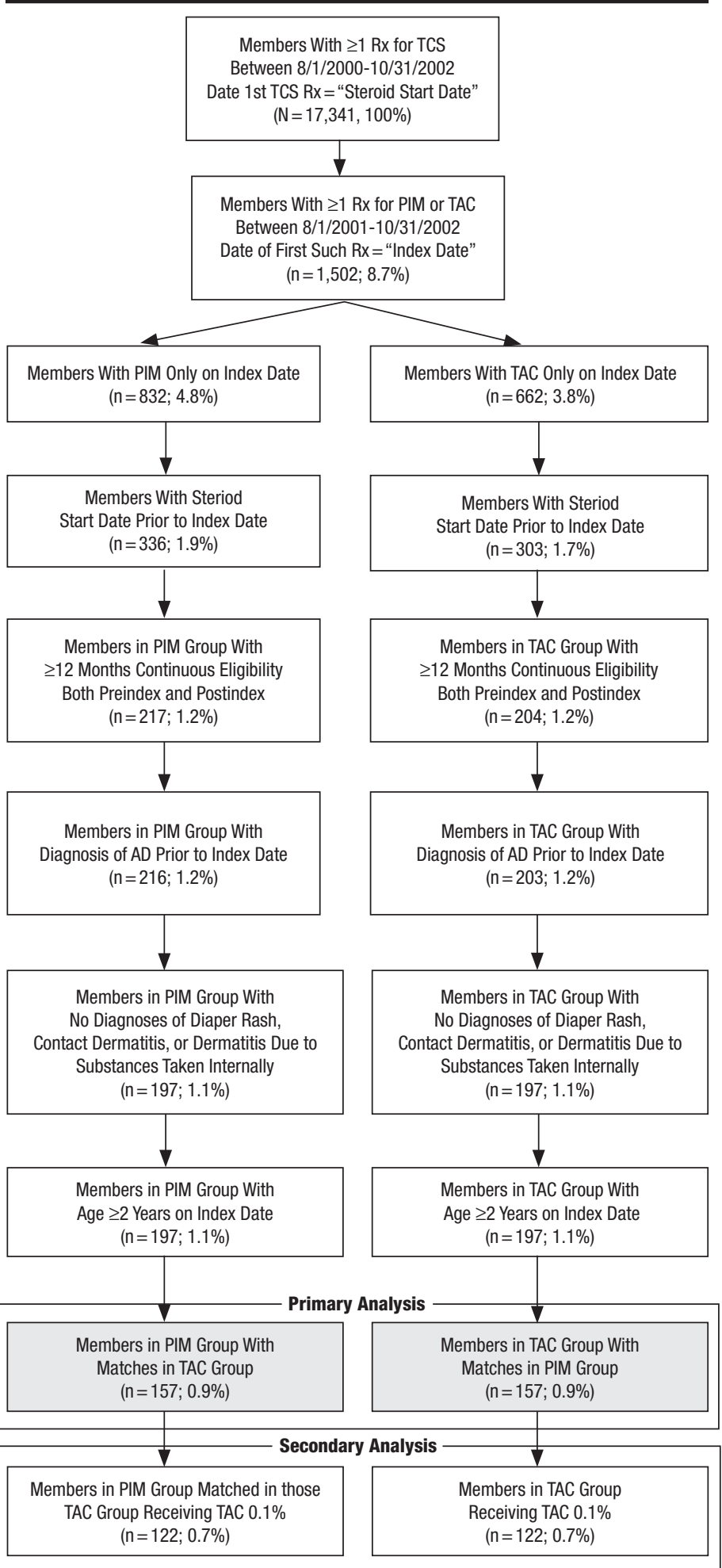

$A D=$ atopic dermatitis; $P I M=$ pimecrolimus; $R x=$ pharmacy claim; $T A C=$ tacrolimus; TCS = topical corticosteroid . 


\section{Administrative Claims Analysis of Utilization and Costs of Care in Health Plan Members With Atopic Dermatitis}

Who Had Prior Use of a Topical Corticosteroid and Who Initiate Therapy With Pimecrolimus or Tacrolimus

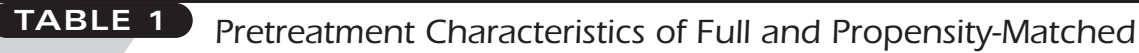

Samples of Pimecrolimus and Tacrolimus Patients*

\begin{tabular}{|c|c|c|c|c|c|c|c|c|c|c|}
\hline \multirow[b]{3}{*}{ Characteristic } & \multicolumn{5}{|c|}{ Full Sample } & \multicolumn{5}{|c|}{ Propensity-Matched Sample } \\
\hline & \multicolumn{2}{|c|}{ Pimecrolimus } & \multicolumn{2}{|c|}{ Tacrolimus } & \multirow[b]{2}{*}{$P$ Value } & \multirow{2}{*}{\multicolumn{2}{|c|}{$\begin{array}{c}\text { Pimecrolimus } \\
\mathrm{N}=157\end{array}$}} & \multirow{2}{*}{\multicolumn{2}{|c|}{$\begin{array}{c}\text { Tacrolimus } \\
\mathrm{N}=157\end{array}$}} & \multirow[b]{2}{*}{$P$ Value } \\
\hline & \multicolumn{2}{|c|}{$\mathrm{N}=197$} & \multicolumn{2}{|c|}{$\mathrm{N}=197$} & & & & & & \\
\hline \multicolumn{11}{|l|}{ Age, years } \\
\hline $2-12$ & 47 & (24) & 59 & (30) & 0.173 & 46 & (29) & 39 & (25) & 0.374 \\
\hline $13-18$ & 17 & (9) & 22 & (11) & 0.399 & 13 & (8) & 18 & (11) & 0.344 \\
\hline$>18$ & 133 & $(68)$ & 116 & (59) & 0.076 & 98 & $(62)$ & 100 & (64) & 0.815 \\
\hline Mean [SD] & 38 & {$[25]$} & 32 & {$[23]$} & 0.022 & 34 & {$[24]$} & 34 & {$[23]$} & 0.842 \\
\hline Male & 80 & $(41)$ & 76 & (39) & 0.680 & 63 & $(40)$ & 59 & (38) & 0.643 \\
\hline South region & 43 & $(22)$ & 35 & (18) & 0.312 & 28 & (18) & 31 & (20) & 0.665 \\
\hline Health maintenence organization & 108 & $(55)$ & 132 & $(67)$ & 0.013 & 98 & $(62)$ & 102 & $(65)$ & 0.639 \\
\hline Other inflammatory skin conditions & 56 & (28) & 44 & $(22)$ & 0.165 & 37 & (24) & 39 & $(25)$ & 0.792 \\
\hline Antihistamine & 103 & $(52)$ & 102 & $(52)$ & 0.920 & 77 & $(49)$ & 75 & $(48)$ & 0.821 \\
\hline Systemic immunosupprresants & 27 & (14) & 31 & (16) & 0.570 & 21 & (13) & 23 & (15) & 0.745 \\
\hline Antistaphylococcal antibiotics & 46 & (23) & 50 & $(25)$ & 0.639 & 38 & (24) & 39 & $(25)$ & 0.896 \\
\hline Nonspecific dermatitis & 136 & (69) & 143 & (73) & 0.438 & 115 & (73) & 118 & $(75)$ & 0.699 \\
\hline \multicolumn{11}{|l|}{ Topical corticosteroids, mean [SD] } \\
\hline \multicolumn{11}{|l|}{ No. of pharmacy claims } \\
\hline Low potency & 0.14 & [0.39] & 0.39 & {$[1.01]$} & 0.002 & 0.17 & {$[0.43]$} & 0.15 & {$[0.51]$} & 0.718 \\
\hline Medium potency & 0.92 & {$[1.58]$} & 1.14 & [1.94] & 0.232 & 1.00 & [1.69] & 0.92 & [1.33] & 0.657 \\
\hline High potency & 1.02 & {$[2.01]$} & 1.48 & {$[2.65]$} & 0.050 & 1.08 & {$[2.17]$} & 1.20 & [1.99] & 0.607 \\
\hline Total & 2.08 & {$[2.45]$} & 3.01 & {$[3.27]$} & 0.002 & 2.25 & [2.65] & 2.28 & {$[2.11]$} & 0.925 \\
\hline \multicolumn{11}{|l|}{ No. of grams } \\
\hline Low potency & 5 & {$[16]$} & 28 & {$[144]$} & 0.025 & 6 & {$[17]$} & 5 & {$[20]$} & 0.856 \\
\hline Medium potency & 78 & {$[228]$} & 88 & {$[211]$} & 0.657 & 73 & {$[178]$} & 69 & {$[166]$} & 0.858 \\
\hline High potency & 49 & {$[103]$} & 77 & {$[157]$} & 0.037 & 52 & {$[110]$} & 63 & {$[125]$} & 0.399 \\
\hline Total & 132 & [249] & 193 & {$[300]$} & 0.029 & 131 & [213] & 138 & {$[208]$} & 0.757 \\
\hline \multicolumn{11}{|l|}{ No. of AD-related visits, mean [SD] } \\
\hline Specialist & 1.19 & {$[1.88]$} & 1.21 & {$[1.21]$} & 0.899 & 1.19 & {$[2.00]$} & 1.19 & [1.12] & 1.000 \\
\hline Nonspecialist & 0.63 & [0.82] & 0.87 & [1.14] & 0.020 & 0.72 & [0.82] & 0.70 & [0.93] & 0.847 \\
\hline All & 1.81 & {$[1.84]$} & 2.05 & {$[1.45]$} & 0.162 & 1.90 & [1.94] & 1.87 & {$[1.21]$} & 0.889 \\
\hline \multicolumn{11}{|l|}{ AD-related costs, $\$$, mean [SD] } \\
\hline Medications & 50 & {$[95]$} & 78 & {$[148]$} & 0.029 & 54 & {$[101]$} & 57 & {$[103]$} & 0.776 \\
\hline Visits & 134 & {$[268]$} & 151 & {$[242]$} & 0.496 & 141 & [292] & 143 & {$[222]$} & 0.939 \\
\hline Total & 184 & [292] & 229 & {$[293]$} & 0.127 & 195 & {$[317]$} & 201 & {$[254]$} & 0.865 \\
\hline
\end{tabular}




\section{Administrative Claims Analysis of Utilization and Costs of Care in Health Plan Members With Atopic Dermatitis Who Had Prior Use of a Topical Corticosteroid and Who Initiate Therapy With Pimecrolimus or Tacrolimus}

the index date; 359 (54\%) of those in the tacrolimus group were excluded for this reason. A total of 394 members met all other study inclusion criteria, including, coincidentally, 197 who had claims for pimecrolimus and 197 who had claims for tacrolimus on the index date. After propensity score matching, there were 157 members in each group.

\section{Pretreatment Characteristics}

The pretreatment characteristics of study subjects for the full and propensity-matched samples are shown in Table 1. Before matching, members receiving pimecrolimus tended to be older than those receiving tacrolimus (mean 38 vs. 32 years, $P=0.022$ ) and were less likely to be enrolled in HMOs (55\% vs. $67 \%$, $P=0.013$ ). Members in the pimecrolimus group received fewer pharmacy claims for topical corticosteroids (mean 2.08 vs. 3.01, $P=0.002$ ) and fewer total grams of topical corticosteroids (mean 132 vs. $193, P=0.029$ ) during the pretreatment period than did those in the tacrolimus group. They also had fewer pretreatment AD-related nonspecialist visits (mean 0.63 vs. $0.87, P=0.020$ ) and lower pretreatment costs of AD-related pharmacy claims (mean $\$ 50$ vs. $\$ 78, P=0.029$ ). The 2 groups were similar in terms of sex, region, pretreatment diagnoses for other skin conditions, and use of antihistamines, systemic immunosuppressives, and antistaphylococcal antibiotics.

After propensity score matching, there were no statistically significant differences in pretreatment characteristics between groups. Mean age was 34 years in both the pimecrolimus and tacrolimus groups $(P=0.842)$. Mean total pharmacy claims for topical corticosteroids during the pretreatment period were 2.25 and 2.28 in the pimecrolimus and tacrolimus groups, respectively $(P=0.925)$. Mean total AD-related costs during the pretreatment period were $\$ 195$ for the pimecrolimus group and $\$ 201$ for the tacrolimus group $(P=0.865)$.

\section{Utilization and Costs During Follow-up}

Measures of utilization and costs during follow-up for the full and propensity-matched samples are shown in Table 2. Before matching, members who received pimecrolimus had fewer total pharmacy claims for topical corticosteroids (mean 1.32 vs. 2.30, $P<0.001$ ) and fewer total grams of topical corticosteroids (mean 82 vs. 193, $P=0.015$ ) during follow-up than had members who received tacrolimus. Pimecrolimus members also were less likely to receive tacrolimus during follow-up than were tacrolimus members to receive pimecrolimus during follow-up (5\% vs. $17 \%, P<0.001)$. Utilization of concomitant medications was similar in the 2 groups. Members receiving pimecrolimus had fewer total AD-related visits (mean 0.84 vs. 1.18, $P=0.024$ ) but not fewer AD-related specialist visits (mean 0.61 vs. 0.85 , $P=0.054$. They also had lower total costs of AD-related medical care (mean $\$ 258$ vs. $\$ 440, P<0.001$ ), primarily due to lower costs of AD medications (mean $\$ 198$ vs. $\$ 349, P<0.001$ ).

Differences between groups in study outcomes during the follow-up were generally attenuated by propensity matching. Nevertheless, even after matching, members in the pimecrolimus group had fewer pharmacy claims for topical corticosteroids during follow-up than had those in the tacrolimus group (mean 1.37 vs. $2.04, P=0.021$ ); this difference was largely due to fewer claims for high-potency topical corticosteroids among pimecrolimus members (mean 0.61 vs. 1.04, $P=0.023$ ). Fewer pimecrolimus members had pharmacy claims for antistaphylococcal antibiotics during follow-up than had the matched tacrolimus members ( $16 \%$ vs. $27 \%, P=0.014)$. Total AD-related costs during follow-up also were lower among propensity-matched members receiving pimecrolimus (mean $\$ 263$ vs. $\$ 361, P=0.012$ ).

\section{Pimecrolimus Versus Tacrolimus 0.1\%}

Of the 197 members who met all criteria for inclusion and initiated therapy with tacrolimus, 144 (74\%) received tacrolimus $0.1 \%$ on the index date. After matching these members to those receiving pimecrolimus, using propensity scores, we found that there were 122 matched pairs of pimecrolimus and tacrolimus $0.1 \%$ members. Results from this analysis (Tables 3 and 4) were generally similar to those comparing pimecrolimus with both formulations of tacrolimus. After matching, members receiving pimecrolimus on their index date had fewer pharmacy claims for high-potency topical corticosteroids during follow-up (mean 0.70 vs. 1.13, $P=0.047$ ), were less likely to switch therapy during followup ( $5 \%$ vs. $16 \%, P=0.006)$, and were less likely to have a pharmacy claim for an antistaphylococcal antibiotic during follow-up (mean $16 \%$ vs. $28 \%, P=0.020$ ) than were those who received tacrolimus $0.1 \%$. Total AD-related expenditures were not significantly different between the 2 groups in the 12 -month follow-up period (mean $\$ 308$ for pimecrolimus versus $\$ 376$ for tacrolimus $[P=0.137])$.

\section{Discussion}

We conducted a retrospective observational comparison of utilization and costs of AD-related medical care in health plan members with $\mathrm{AD}$ who received topical corticosteroids and subsequently initiated therapy with pimecrolimus or tacrolimus. After attempting to control for differences between groups using propensity matching on baseline clinical characteristics and pretreatment utilization of AD-related medical care, we found that pimecrolimus members had fewer pharmacy claims for topical corticosteroids, were less likely to switch therapy, were less likely to receive antistaphylococcal antibiotics, and had lower costs of AD-related medical care during follow-up than had corresponding tacrolimus members.

A recent pooled analysis of 413 adults and 650 pediatric patients participating in 3 randomized studies found that tacrolimus was more effective than pimecrolimus, based on a variety of measures. ${ }^{34} \mathrm{~A}$ randomized, investigator-blinded 


\section{Administrative Claims Analysis of Utilization and Costs of Care in Health Plan Members With Atopic Dermatitis}

Who Had Prior Use of a Topical Corticosteroid and Who Initiate Therapy With Pimecrolimus or Tacrolimus

\section{TABLE 2 Utilization and Costs of AD-Related Care During Follow-up for Full and \\ Propensity-Matched Samples of Pimecrolimus and Tacrolimus Patients*}

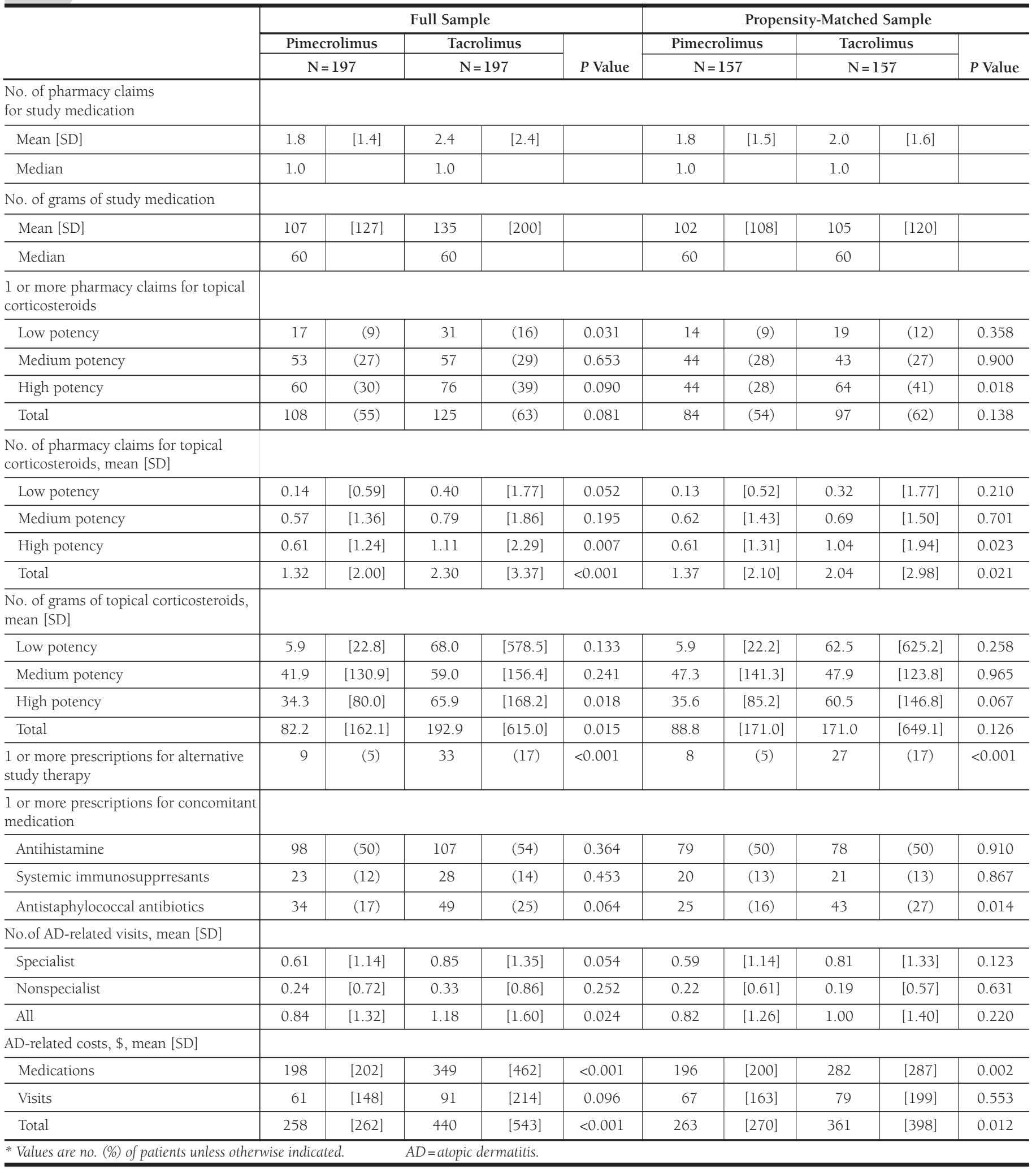




\section{Administrative Claims Analysis of Utilization and Costs of Care in Health Plan Members With Atopic Dermatitis Who Had Prior Use of a Topical Corticosteroid and Who Initiate Therapy With Pimecrolimus or Tacrolimus}

TABLE 3 Pretreatment Characteristics of Propensity-Matched Sample of Pimecrolimus and Tacrolimus $0.1 \%$ Patients*

\begin{tabular}{|c|c|c|c|c|c|}
\hline \multirow[b]{2}{*}{ Characteristic } & \multicolumn{2}{|c|}{ Pimecrolimus } & \multicolumn{2}{|c|}{ Tacrolimus $0.1 \%$} & \multirow[b]{2}{*}{$P$ Value } \\
\hline & \multicolumn{2}{|c|}{$\mathrm{N}=122$} & \multicolumn{2}{|c|}{$\mathrm{N}=122$} & \\
\hline \multicolumn{6}{|l|}{ Age, years } \\
\hline $2-12$ & 22 & (18) & 19 & (16) & 0.607 \\
\hline $13-18$ & 10 & (8) & 16 & (13) & 0.213 \\
\hline$>18$ & 90 & $(74)$ & 87 & $(71)$ & 0.667 \\
\hline Mean [SD] & 41 & {$[24]$} & 39 & {$[22]$} & 0.431 \\
\hline Male & 48 & (39) & 44 & (36) & 0.597 \\
\hline South region & 23 & (19) & 22 & (18) & 0.869 \\
\hline Health maintenence organization & 76 & $(62)$ & 75 & $(61)$ & 0.895 \\
\hline Other inflammatory skin conditions & 33 & $(27)$ & 32 & $(26)$ & 0.885 \\
\hline Antihistamine & 58 & $(48)$ & 57 & $(47)$ & 0.898 \\
\hline Systemic immunosupprresants & 19 & (16) & 19 & (16) & 1.000 \\
\hline Antistaphylococcal antibiotics & 36 & (30) & 34 & $(28)$ & 0.777 \\
\hline Nonspecific dermatitis & 95 & (78) & 91 & $(75)$ & 0.547 \\
\hline \multicolumn{6}{|l|}{ Topical corticosteroids, mean [SD] } \\
\hline \multicolumn{6}{|l|}{ No. of pharmacy claims } \\
\hline Low potency & 0.15 & {$[0.42]$} & 0.18 & {$[0.59]$} & 0.617 \\
\hline Medium potency & 1.04 & {$[1.86]$} & 0.87 & {$[1.38]$} & 0.414 \\
\hline High potency & 1.35 & {$[2.38]$} & 1.39 & {$[2.21]$} & 0.889 \\
\hline Total & 2.54 & [2.88] & 2.44 & [2.48] & 0.775 \\
\hline \multicolumn{6}{|l|}{ No. of grams } \\
\hline Low potency & 6 & [19] & 8 & [26] & 0.538 \\
\hline Medium potency & 98 & [279] & 78 & [198] & 0.516 \\
\hline High potency & 66 & {$[121]$} & 67 & {$[117]$} & 0.951 \\
\hline Total & 170 & [299] & 152 & [231] & 0.611 \\
\hline \multicolumn{6}{|l|}{ No. of AD-related visits, mean [SD] } \\
\hline Specialist & 1.34 & {$[2.07]$} & 1.30 & [1.24] & 0.822 \\
\hline Nonspecialist & 0.71 & {$[0.87]$} & 0.66 & {$[0.82]$} & 0.596 \\
\hline All & 2.04 & {$[2.01]$} & 1.93 & [1.25] & 0.620 \\
\hline \multicolumn{6}{|l|}{ AD-related costs, $\$$, mean $[\mathrm{SD}]$} \\
\hline Medications & 65 & {$[112]$} & 57 & [95] & 0.512 \\
\hline Visits & 159 & [319] & 149 & [236] & 0.768 \\
\hline Total & 224 & [346] & 205 & [265] & 0.624 \\
\hline
\end{tabular}

* Values are no. (\%) of patients unless otherwise indicated.

$A D=$ atopic dermatitis.

study of pimecrolimus and tacrolimus ointment $0.03 \%$ in 141 pediatric patients reported similar efficacy with pimecrolimus and tacrolimus but superior local tolerability and more favorable ratings of product characteristics with pimecrolimus. ${ }^{33}$ While we did not examine these specific outcomes in our study, it is possible that the differences in outcomes that we observed 


\section{Administrative Claims Analysis of Utilization and Costs of Care in Health Plan Members With Atopic Dermatitis Who Had Prior Use of a Topical Corticosteroid and Who Initiate Therapy With Pimecrolimus or Tacrolimus}

\section{TABLE 4 Utilization and Costs of AD-Related Care During Follow-up for}

Propensity-Matched Sample of Pimecrolimus and Tacrolimus $0.1 \%$ Patients*

\begin{tabular}{|c|c|c|c|c|c|}
\hline \multirow[b]{3}{*}{ No. of pharmacy claims for study medication } & \multirow{2}{*}{\multicolumn{2}{|c|}{$\begin{array}{c}\text { Pimecrolimus } \\
\mathrm{N}=122\end{array}$}} & \multirow{2}{*}{\multicolumn{2}{|c|}{$\begin{array}{c}\text { Tacrolimus } 0.1 \% \\
\mathrm{~N}=122\end{array}$}} & \multirow{3}{*}{$P$ Value } \\
\hline & & & & & \\
\hline & \multirow[b]{2}{*}{1.9} & \multirow{3}{*}[1.7]{} & \multirow{3}{*}{$\begin{array}{l}2.0 \\
1.0\end{array}$} & \multirow{3}{*}[1.7]{} & \\
\hline Mean [SD] & & & & & \\
\hline Median & 1.0 & & & & \\
\hline \multicolumn{6}{|l|}{ No. of grams of study medication } \\
\hline Mean $[S D]$ & 125 & {$[144]$} & 108 & [111] & \\
\hline Median & 95 & & 60 & & \\
\hline \multicolumn{6}{|l|}{1 or more pharmacy claims for topical corticosteroids } \\
\hline Low potency & 13 & (11) & 15 & (12) & 0.688 \\
\hline Medium potency & 35 & (29) & 33 & $(27)$ & 0.775 \\
\hline High potency & 37 & (30) & 57 & $(47)$ & 0.009 \\
\hline Total & 66 & (54) & 79 & $(65)$ & 0.090 \\
\hline \multicolumn{6}{|c|}{ No. of pharmacy claims for topical corticosteroids, mean [SD] } \\
\hline Low potency & 0.16 & [0.58] & 0.34 & [1.96] & 0.352 \\
\hline Medium potency & 0.74 & [1.64] & 0.58 & [1.31] & 0.413 \\
\hline High potency & 0.70 & [1.42] & 1.13 & [1.88] & 0.047 \\
\hline Total & 1.61 & [2.36] & 2.05 & [2.98] & 0.200 \\
\hline \multicolumn{6}{|l|}{ No. of grams of topical corticosteroids, mean [SD] } \\
\hline Low potency & 7 & {$[25]$} & 73 & {$[708]$} & 0.310 \\
\hline Medium potency & 54 & {$[156]$} & 38 & [96] & 0.343 \\
\hline High potency & 43 & {$[97]$} & 66 & [153] & 0.171 \\
\hline Total & 104 & [195] & 176 & {$[723]$} & 0.289 \\
\hline 1 or more prescriptions for alternative study therapy & 6 & (5) & 19 & (16) & 0.006 \\
\hline \multicolumn{6}{|l|}{1 or more prescriptions for concomitant medication } \\
\hline Antihistamine & 61 & (50) & 61 & $(50)$ & 1.000 \\
\hline Systemic immunosupprresants & 16 & (13) & 21 & (17) & 0.372 \\
\hline Antistaphylococcal antibiotics & 19 & (16) & 34 & (28) & 0.020 \\
\hline \multicolumn{6}{|l|}{ No. of AD-related visits, mean [SD] } \\
\hline Specialist & 0.69 & [1.26] & 0.87 & {$[1.24]$} & 0.261 \\
\hline Nonspecialist & 0.28 & [0.83] & 0.20 & {$[0.54]$} & 0.360 \\
\hline All & 0.96 & {$[1.47]$} & 1.07 & [1.35] & 0.555 \\
\hline \multicolumn{6}{|l|}{ AD-related costs, $\$$, mean [SD] } \\
\hline Medications & 231 & [230] & 288 & [273] & 0.081 \\
\hline Visits & 76 & {$[177]$} & 88 & {$[216]$} & 0.649 \\
\hline Total & 308 & [298] & 376 & {$[408]$} & 0.137 \\
\hline
\end{tabular}

* Values are no. (\%) of patients unless otherwise indicated.

$A D=$ atopic dermatitis.

were due to differences in tolerability and patient perceptions regarding product characteristics that have been reported elsewhere. ${ }^{33}$

\section{Limitations}

First and foremost, this was a retrospective, observational study using health insurance administrative claims data. Such studies 


\section{Administrative Claims Analysis of Utilization and Costs of Care in Health Plan Members With Atopic Dermatitis Who Had Prior Use of a Topical Corticosteroid and Who Initiate Therapy With Pimecrolimus or Tacrolimus}

are useful for examining outcomes in "real world" settings where patient selection and treatment conditions are more inclusive than are typically done in tightly controlled randomized controlled trials. Because treatment with pimecrolimus or tacrolimus was not randomly assigned, it is possible that our findings were due to confounding factors that were not accounted for in our analyses. Of particular importance is the issue of disease severity. Pimecrolimus is approved for use in the United States in patients with mild-to-moderate $\mathrm{AD},{ }^{29}$ whereas tacrolimus is approved for use in patients with moderate-to-severe AD. ${ }^{30}$ One would expect, therefore, that members receiving pimecrolimus would have less severe disease than those receiving tacrolimus. Indeed, we found that members in the pimecrolimus group utilized less topical corticosteroid and had fewer visits in the pretreatment period than had those in the tacrolimus group. Also, members who received pimecrolimus were less likely to have received topical corticosteroids prior to therapy initiation. Although we attempted to control for these differences by selecting patients who received prior steroid therapy and by using propensity-score matching, we lacked information on disease severity and were unable to control for it directly in our analysis.

Second, since we required AD patients in this study to have received at least 1 prior pharmacy claim for a topical steroid, we may have excluded many patients who were using only overthe-counter (OTC) topical steroids because OTC topical steroid use was not measured. We also do not know the extent of OTC topical steroid use during the 12-month follow-up period.

Third, our sample size was relatively small, which precluded subgroup analyses based on baseline characteristics such as age, sex, level of pretreatment utilization of steroids, or dosage or intensity of treatment with pimecrolimus or tacrolimus.

Fourth, because our sample was drawn from members in a variety of health plans offered by a single large U.S. health benefits company, our findings may not be generalizable to patients in other countries, regions, or types of health plans.

Fifth, we did not evaluate a control group of AD patients who received only corticosteroids and neither tacrolimus nor pimecrolimus. Although this group of patients might have provided additional information about the value of tacrolimus or pimecrolimus compared with corticosteroids, it is likely that any comparison would be confounded because receipt of topical calcineurin inhibitors is very probably highly correlated with response to topical steroid therapy.

\section{Conclusions}

We found that compared with health plan members who used tacrolimus, members with $\mathrm{AD}$ who used pimecrolimus and had previously received at least 1 topical corticosteroid prescription received fewer pharmacy claims for topical corticosteroids, were less likely to receive antistaphylococcal antibiotics, and had lower total costs of AD-related medical care. Further research using validated measures of disease severity is needed to confirm the results of this observational study.

\section{Authors}

THOMAS E. DELEA, MSIA, is a senior research consultant, Policy Analysis Inc. (PAI), Brookline, Massachusetts; MANJUSHA GOKHALE, $M A$, is senior programmer/analyst, Center for Clinical Excellence, Brigham and Women's Hospital, Boston, MA. (at the time of this study, she was a programmer analyst at PAI); CHARLES MAKIN, MS, is a senior clinical analyst, HealthCore, Inc., Wilmington Delaware (at the time of this study, he was a pharmacoeconomics and outcomes research fellow, Humana Outcomes Research Center, Humana Inc., Louisville, Kentucky); MOHAMED A. HUSSEIN, MSCS, MSPh, is an associate director, outcomes research and pharmacoeconomics, ValueMedics Research, LLC, Falls Church, Virginia (at the time of this study, he was a statistical analyst, Humana Outcomes Research Center, Humana Inc., Louisville, Kentucky); JULIE VANDERPOEL, PharmD, MPA, is a clinical scientist, Centocor, Louisville, Kentucky (at the time of this study, she was a senior project manager, Humana Outcomes Research Center, Humana Inc., Louisville, Kentucky); TIMOTHY SANDMAN, MBA, is an outcomes analyst, Humana Outcomes Research Center, Humana Inc., Louisville, Kentucky; JANE CHANG, MPH, is a manager, Health Economics \& Outcomes Research (HEEOR), Novartis Pharmaceuticals Corporation, East Hanover, New Jersey (at the time of this study, she was a HEEOR fellow at Duke University, Durham, North Carolina, and Novartis Pharmaceuticals Corporation, East Hanover, New Jersey); JENNIFER SUNG, PharmD, is director, HEEOR, Novartis Pharmaceuticals Corporation, East Hanover, New Jersey; PAUL PINKSTON, PharmD, is director, managed markets - national accounts, Novartis Pharmaceuticals Corporation, East Hanover, New Jersey; DOUGLAS GAUSE, PhD, is assistant director, HEEOR, Novartis Pharmaceuticals Corporation, East Hanover, New Jersey; J. MARK JACKSON, MD, is assistant clinical professor of medicine and dermatology, University of Louisville, Division of Dermatology, Kentucky.

AUTHOR CORRESPONDENCE: Thomas E. Delea, MSIA, Senior Research Consultant, Policy Analysis Inc., (PAI), Four Davis Ct., Brookline, MA 02445. Tel: (617) 232-4400; Fax: (617) 232-1155; E-mail: tdelea@pai2.com

\section{DISCLOSURES}

Funding for this study was provided by Novartis Pharmaceuticals Corporation and was obtained by author Thomas E. Delea. Authors Jane Chang, Jennifer Sung, Paul Pinkston, and Douglas Gause are employed by Novartis Pharmaceuticals Corporation. The authors disclose the following: Delea is employed by Policy Analysis Inc. (PAI), which has received research support and consulting fees from Novartis; author Manjusha Gokhale was employed by PAI, which has received research support and consulting fees from Novartis; author Charles Makin is employed by Health Core, Inc., which provides contract research and consulting services to the pharmaceutical industry; author Mohamed A. Hussein is employed by ValueMedics Research, LLC, which has received research and consulting fees from Novartis; author J. Mark Jackson has received honoraria, consulting fees, and research support from Novartis. At the time this study was conducted, Hussein and authors Julie Vanderpoehl and Timothy Sandman were employees of the Outcomes Research Center, Humana Inc., which provides research services to a variety of pharmaceutical companies.

Results of this study were presented, in part, at the American Academy of Dermatology 2005 Annual Meeting, June 5-8, 2004, New Orleans, Louisiana, and the 17th Annual Meeting and Showcase of the Academy of Managed Care Pharmacy, April 20-23, 2005, Denver, Colorado. Delea served as principal 


\section{Administrative Claims Analysis of Utilization and Costs of Care in Health Plan Members With Atopic Dermatitis Who Had Prior Use of a Topical Corticosteroid and Who Initiate Therapy With Pimecrolimus or Tacrolimus}

author of the study. Study concept and design were contributed by Delea, Sung, Pinkston, and Gause. Data collection was the work of Makin, Hussein, Vanderpoel, and Sandman; data interpretation was primarily the work of Delea and Gokhale, with input from the coauthors. Writing of the manuscript was the work of Delea, Gokhale, Chang, and Jackson; its revision was primarily the work of Gokhale and Delea, with input from the coauthors.

\section{REFERENCES}

1. Leung DY, Bieber T. Atopic dermatitis. Lancet. 2003;361:151-60.

2. Schultz-Larson F, Hanifin JM. Epidemiology of atopic dermatitis. Immunol Allergy Clin North Am. 2002;22:1-24.

3. Taylor B, Wadsworth J, Wadsworth M, Peckham C. Changes in the reported prevalence of childhood eczema since the 1939-45 war. Lancet. 1984;2:1255-57.

4. Kiebert G, Sorensen SV, Revicki D, et al. Atopic dermatitis is associated with a decrement in health-related quality of life. Int J Dermatol. 2002;41(3):151-58.

5. Lundberg L, Johannesson M, Silverdahl M, Hermansson C, Lindberg M. Health-related quality of life in patients with psoriasis and atopic dermatitis measured with SF-36, DLQI and a subjective measure of disease activity. Acta Derm Venereol. 2000;80(6):430-34.

6. Lundberg L, Johannesson M, Silverdahl M, Hermansson C, Lindberg M. Quality of life, health-state utilities and willingness to pay in patients with psoriasis and atopic eczema. Br J Dermatol. 1999;141(6):1067-75.

7. Ellis CN, Drake LA, Prendergast MM, et al. Cost of atopic dermatitis and eczema in the United States. J Am Acad Dermatol. 2002;46:361-70.

8. Fivenson D, Arnold RJ, Kaniecki DJ, Cohen JL, Frech F, Finlay AY. The effect of atopic dermatitis on total burden of illness and quality of life on adults and children in a large managed care organization. J Manag Care Pharm. 2002;8(5):333-42. Available at: http://www.amcp.org/data/jmcp/Research-333342.pdf. Accessed April 21, 2007.

9. Johnson N, Kahler K. Total cost of care for patients with pediatric atopic dermatitis. Poster presented at: the American Academy of Dermatology 60th Annual Meeting; February 22-27, 2002; New Orleans, LA. Poster 121.

10. Su JC, Kemp AS, Varigos GA, Nolan TM. Atopic eczema: its impact on the family and financial cost. Arch Dis Child. 1997;76:159-62.

11. Lapidus CS, Schwarz DF, Honig PJ. Atopic dermatitis in children: who cares? who pays? J Am Acad Dermatol. 1993;28:699-703

12. Fisher DA. Adverse effects of topical corticosteroid use. West J Med. 1995; 162:123-26

13. Kristmundsdottir F, David TJ. Growth impairment in children with atopic eczema. J R Soc Med. 1987;80:9-12

14. Bode HH. Dwarfism following long-term topical corticosteroid therapy. JAMA. 1980;244:813-14

15. Ellison JA, Patel L, Ray DW, David TJ, Clayton PE. Morning plasma cortisol levels in infants treated with topical fluorinated glucocorticosteroids. Pediatrics. 1980;65:103-06

16. Paller AS, McAlister RO, Doyle JJ, Jackson A. Perceptions of physicians and pediatric patients about atopic dermatitis, its impact, and its treatment. Clin Pediatr. 2002;41:323-32.

17. Charman C, Morris AD, Williams HC. Topical corticosteroid phobia in patients with atopic dermatitis. Br J Dermatol. 2000;142:931-36.

18. Weinberg JM. Formulary review of therapeutic alternatives for atopic dermatitis: focus on pimecrolimus. J Manag Care Pharm. 2005:11(1):56-64 Available at: http://www.amcp.org/data/jmcp/Form_Manag_56-64.pdf. Accessed April 21, 2007

19. Eichenfield LF, Lucky AW, Boguniewicz M, et al. Safely and efficacy of pimecrolimus (ASM 981) cream 1\% in the treatment of mild and moderate atopic dermatitis in children and adolescents. J Am Acad Dermatol. 2002;46(4):495-504

20. Meurer M, Folster-Holst R, Wozel G, et al; CAM-DE-01 Study Group. Pimecrolimus cream in the long-term management of atopic dermatitis in adults: a six-month study. Dermatology. 2002;205(3):271-77.
21. Hanifin JM, Ling MR, Langley R, Breneman D, Rafal E. Tacrolimus ointment for treatment of atopic dermatitis in adult patients: Part I, efficacy. J Am Acad Dermatol. 2001;44(suppl 1):S28-S38.

22. Paller A, Eichenfield LF, Leung DY, Stewart D, Appell M. A 12-week study of tacrolimus ointment for the treatment of atopic dermatitis in pediatric patients. J Am Acad Dermatol. 2001;44(suppl 1):S47-S57.

23. Boguniewicz M, Fiedler VC, Raimer S, et al. Pediatric Tacrolimus Study Group. A randomized vehicle-controlled trial of tacrolimus ointment for treatment of atopic dermatitis in children. J Allergy Clin Immunol. 1998;102 (4 pt 1);555-57.

24. Ruzicka T, Bieber T, Schopf E, et al. A short-term trial of tacrolimus ointment for atopic dermatitis. N Engl J Med. 1997;337:816-21.

25. Kapp A, Papp K, Bingham A, et al. Flare Reduction in Eczema with Elidel (Infants) Multicenter Investigator Study Group. Long-term management of atopic dermatitis in infants with topical pimecrolimus, a nonsteroidal anti-inflammatory drug. J Allergy Clin Immunol. 2002;110(2):277-84.

26. Wahn U, Bos JD, Goodfield M, et al. Flare Reduction in Eczema with Elidel (Children) Multicenter Investigator Study Group. Efficacy and safety of pimecrolimus cream in the long-term management of atopic dermatitis in children. Pediatrics. 2002;110(1 pt 1):e2.

27. Soter NA, Fleischer AB, Webster GF, Monroe E, Lawrence I. Tacrolimus ointment for the treatment of atopic dermatitis in adult patients: Part II, safety. J Am Acad Dermatol. 2001;44(suppl 1):S39-S46.

28. Meingassner JG, Fahrngruber H, Bavandi A. Pimecrolimus inhibits the elicitation phase but does not suppress the sensitization phase in murine contact hypersensitivity, in contrast to tacrolimus and cyclosporine A. J Invest Dermatol. 2003;121(1):77-80. Erratum: J Invest Dermatol. 2003;121(5):1231.

29. Elidel [prescribing information]. Available at: http://www.pharma. us.novartis.com/product/pi/pdf/elidel.pdf. Accessed June 2006.

30. Protopic [prescribing information]. Available at: http://www.astellas. us/docs/protopic.pdf. Accessed June 2006.

31. FDA Public Health Advisory. Available at: http://www.fda.gov/cder/drug/ advisory/elidel_protopic.htm. Accessed June 2006.

32. Iskedjian M, Piwko C, Shear NH, Langley RG, Einarson TR. Topical calcineurin inhibitors in the treatment of atopic dermatitis: a meta-analysis of current evidence. Am J Clin Dermatol. 2004;5(4):267-79.

33. Kempers S, Boguniewicz M, Carter E, et al. A randomized investigatorblinded study comparing pimecrolimus cream $1 \%$ with tacrolimus ointment $0.03 \%$ in the treatment of pediatric patients with moderate atopic dermatitis. J Am Acad Dermatol. 2004;51(4):515-25.

34. Paller AS, Lebwohl M, Fleischer AB, et al. Tacrolimus ointment is more effective than pimecrolimus cream for patients with a similar safety profile in the treatment of atopic dermatitis: results from 3 randomized, comparative studies. J Am Acad Dermatology. 2005;52(5):810-22.

35. Chang J, Sung J. Health plan budget impact analysis for pimecrolimus. J Manag Care Pharm. 2005;11(1):66-73. Available at: http://www.amcp.org/ data/jmcp/Form_Manag_66-73.pdf. Accessed April 21, 2007.

36. Ellis CN, Kahler KH, Grueger J, Chang J. Cost effectiveness of management of mild-to-moderate atopic dermatitis with $1 \%$ pimecrolimus cream in children and adolescents 2-17 years of age. Am J Clin Dermatol. 2006;7(2):133-39.

37. Drug Facts and Comparisons 2004. Chicago, IL: Wolters Kluwer Health; 2004.

38. Rosenbaum P, Rubin D. The central role of the propensity score in observational studies for causal effects. Biometrika. 1983;70:41-55.

39. Rubin DB. Estimating causal effects from large datasets using propensity scores. Ann Intern Med. 1997;127:757-63.

40. Parsons L. Reducing bias in a propensity score matched-pair sample using greedy matching techniques. Proceedings of the 26th Annual SAS Users Group International Conference. Cary, NC: SAS Institute; 2001:214-26. Available at: http://support.sas.com/usergroups/sugi/proceedings/. Accessed April 2005. 